\title{
Diferencias entre mujeres y hombres en la asociación entre la salud autopercibida y la mortalidad en las edades adultas en Europa
}

Sex differences in the association between Self-perceived health and mortality at adult ages in Europe

\author{
JoRDI GuMÀ \\ Universidad Pompeu Fabra \\ Amand Blanes \\ Universidad Autónoma de Barcelona \\ jordi.guma@upf.edu (ESPAÑA)
}

Recibido: 27.072016

Aceptado: 21.12 .2017

\section{RESUMEN}

Objetivo: Explorar el patrón por edad de la relación entre el indicador de salud autopercibida y la mortalidad en las edades posteriores a la juventud (35-79) para hombres y mujeres en seis países europeos con trayectorias de morbilidad diferenciadas: Alemania, Francia, España, Italia, Polonia y Hungría.

Métodos y datos: Descripción de los patrones tanto de la prevalencia de mala salud autopercibida como de la diferencia entre las transformaciones logarítmicas de las probabilidades de morir y de la prevalencia de mala salud en los años 2005 y 2009. Los datos proceden de la encuesta sobre Condiciones de Vida en la Unión Europea (EU-SILC) para la salud autopercibida, y de la Human Mortality Database (HMD) para la mortalidad.

Resultados: Ambos indicadores muestran un patrón creciente por edad aunque el valor relativo de este incremento no es igual para la mortalidad y para la mala salud. La prevalencia de mala salud autopercibida aumenta con la edad con una intensidad menor que la mortalidad en ambos sexos en todos los países analizados. Este cambio en la relación entre ambos indicadores con la edad muestra valores similares entre mujeres y hombres, menos en el caso de Polonia y Hungría.

Conclusiones: El cambio en la relación entre salud percibida y mortalidad con la edad se explicaría mediante la normalización por parte del individuo de la 
propia morbilidad. El diferente cambio en esta relación entre mujeres y hombres parece deberse a mayores niveles de desigualdad de género en aquellos países donde se observa esta diferencia, aunque estos resultados deberán comprobarse futuros trabajos.

\title{
PALABRAS CLAVE
}

Salud autopercibida; mortalidad; Europa; EU-SILC.

\begin{abstract}
Goal: To explore the age pattern of the relationship between self-perceived health and mortality at ages beyond youth (35-79) for men and women in six European countries with different with different patterns of morbidity: Germany, France, Spain, Italy, Poland and Hungary.

Methods and data sources: Descriptive analysis of the patterns of both the prevalence of poor self-perceived health and the difference between the logarithmic transformations of the mortality probabilities and the prevalence of poor health in 2005 and 2009. The data about self-perceived health come from the European Union statistics on income and living conditions (EU-SILC), whereas mortality data come from Human Mortality Database (HMD).

Results: Both indicators show a growing pattern by age though the relative value of this increase is not equal in mortality and poor self-perceived health. Poor self-perceived health prevalence rises by age with a lower intensity than mortality for both sexes in all the analysed countries. This change by age in the relationship between both health outcomes shows similar values for women and men, with the exception of Poland and Hungary

Conclusions: The change in the relationship by age between self-perceived health and mortality would be explained by the process of standardization of individual's morbidity. Different age changes between sexes seem to be related with higher levels of gender inequalities in countries where this difference is observed, though this must be confirmed in future research.
\end{abstract}

\section{KEY WORDS}

Self-perceived health; mortality; Europe; EU-SILC.

\section{INTRODUCCIÓN}

La salud autopercibida, indicador perteneciente a la dimensión subjetiva de la salud, ha sido frecuentemente utilizada para aproximar el perfil de morbilidad de las poblaciones (Idler y Benyamini, 1997; Benyamini et al., 2003; Singh- 
Manoux et al., 2006). Sin embargo, aún no se tiene la certeza de qué es lo que realmente mide (estado o evolución de la salud objetiva, etc.), o cuáles son los factores sociodemográficos que la condicionan (Jylhä, 2009). En este sentido, diversos autores han explorado la salud autopercibida comparándola con otros indicadores clásicos sobre salud como la mortalidad. Este indicador de naturaleza demográfica, que ha sido de gran utilidad en el pasado ante la ausencia de otros indicadores, sigue siendo referencia en los estudios a nivel poblacional, acumulando de manera sostenida un amplio conocimiento sobre su patrón por edad y sexo (Vallin et al., 2001).

La mayoría de los trabajos que han analizado la relación entre salud autopercibida y mortalidad han encontrado una alta asociación entre ambas a nivel tanto agregado como individual. De este modo, la salud subjetiva parece predecir la supervivencia de los individuos a corto y medio plazo tanto en las edades adultas (Singh-Manoux et al., 2007; Tamayo-Fonseca et al, 2013) como en las avanzadas, donde se concentran las ganancias en esperanza de vida en los países occidentales (Mossey y Shapiro, 1982; Quesnel-Vallée, 2007). Sin embargo, existen trabajos centrados en poblaciones en edades avanzadas que han mostrado que el nivel de asociación entre ambos indicadores decrece cuanto mayor es la edad de los individuos (Franks et al., 2003; Woo y Zajacova, 2014), relativizando de este modo la creencia general de que la salud autopercibida y la mortalidad muestran patrones paralelos en todos los grupos etarios.

Una posible razón para explicar esas discrepancias radica en la diferente naturaleza de ambos indicadores: subjetiva en el caso de la salud autopercibida y objetiva en el de la mortalidad. En otras palabras, mientras que la mortalidad es muy fácil de medir, en el caso de la salud tenemos que confiar en la evaluación subjetiva individual. Precisamente la naturaleza subjetiva de la salud autopercibida ha originado un rico debate sobre los factores que influyen en la percepción de la propia salud. Por ejemplo, Idler y Benyamini (1997), en una revisión de veintisiete trabajos sobre este indicador mostraron que la percepción en el corto plazo estaba relacionada con la evaluación reciente de las capacidades del individuo, apuntando a una relación predictiva de la dimensión subjetiva de la salud sobre la objetiva. Sin embargo, esta capacidad predictiva está altamente condicionada por el conocimiento que se tenga sobre la propia salud, el cual ha mostrado depender de diversos factores sociodemográficos entre los cuales destacan el sexo y el nivel educativo. Las diferencias entre mujeres y hombres tienen su origen en la construcción social de la concepción de la feminidad y masculinidad, siendo las mujeres las que muestran una mayor preocupación por su estado de salud mientras que los hombres, al contrario, se muestran más reacios al considerarlo como un síntoma de debilidad (Idler, 2003; Oksuzyan et al., 2018). En cuanto a los niveles educativos, niveles más altos no están sólo vinculados a niveles socioeconómicos mayores sino que además están asociados a un mayor conocimiento tanto de los síntomas que permiten una identificación precoz de los problemas así como de salud como de los mecanismos propios de cada país para hacer un uso más óptimo de los recursos sanitarios (Pongiglione y Sabater, 2016). Todo lo anterior apunta a la existencia de una serie de factores 
que pueden llevar a evaluaciones diferentes de la propia salud incluso en situaciones de morbilidad similares. Para poder entender mejor la complejidad de la salud autopercibida como indicador de salud, Jylhä (2009) propuso un modelo conceptual unificado en el cual se apuntan tres contextos claves por su influencia sobre la percepción de los individuos: la biografía individual de salud; los grupos de referencia tomados para esta evaluación (cómo estoy respecto a mis iguales); y las convenciones culturales que condicionan la respuesta dentro de la escala de medida. En cuanto al primer y tercer contexto, definidos a nivel macro, estarían determinados en gran medida por el país o el entorno. Diversos trabajos ya apuntaron diferencias en los perfiles de salud entre países a partir de la salud autopercibida. Concretamente, los países del este y del sur de Europa mostraron peores niveles de salud autopercibida en comparación con los del centro y del norte (Carlson, 1998; Knesebeck, 2006). Las razones que podrían explicar estas diferencias son: los diferentes regímenes de mortalidad de cada país en la primera mitad del siglo XX; el impacto sobre la salud de los modelos de estado del bienestar; o la influencia de factores culturales tanto en los hábitos y comportamientos como en la propia declaración de la salud (Olsen y Dahl, 2007; Eikemo et al., 2008).

En cuanto al segundo contexto apuntado por Jylhä, el grupo de referencia tomado en la evaluación de la propia salud, este varía por edad para cada individuo dependiendo de su etapa vital. La evaluación constante de la propia salud a lo largo del tiempo conlleva que se produzca un reajuste de los criterios de evaluación a nivel tanto individual como contextual (Singh-Manoux, 2006; Oksuzyan et al., 2009). A nivel individual se produce un proceso de normalización o aceptación de la morbilidad y la discapacidad mediante la adaptación de la actividad cotidiana a los límites impuestos por la propia salud, siempre y cuando no se produzcan episodios de empeoramiento significativos en el corto plazo (Gumà y Cámara, 2014). A nivel contextual, la normalización se produce a medida que el perfil de salud del individuo se muestra más similar al de la mayoría de sus coetáneos que conforman su marco de referencia.

Pero además, para entender la posible relación entre la salud autopercibida y la mortalidad se debe tener en cuenta que no siempre morbilidad y mortalidad van de la mano. En este sentido, un buen ejemplo a nivel poblacional se encuentra en la "paradoja de salud y mortalidad" entre sexos, basada en el hecho que las mujeres muestran niveles más bajos de mortalidad respecto de sus coetáneos masculinos, aunque ellas declaran tener peores valores de salud tanto subjetiva como objetiva, siendo mayor esta diferencia con la edad (Oksuzyan et al., 2010; Sanderson y Scherbov, 2017). Precisamente ha sido el diferente perfil de morbilidad según sexo la explicación con un mayor consenso entre los diversos autores para entender esta paradoja. El perfil de las mujeres se caracteriza por una mayor prevalencia de enfermedades crónicas y de largo recorrido como las de naturaleza musculoesquelética o mental, mientras que los hombres presentan una mayor propensión a sufrir enfermedades de menor recorrido pero mayor letalidad como sería el ejemplo de las de tipo cardiovascular (Oksuzyan et al., 2008; Norris et al., 2010). 
En cuanto a la mortalidad en el caso concreto de los países europeos, su evolución a lo largo del s. XX y primeras décadas del s. XXI ha mostrado diferencias según grandes grupos geográficos (centro, este y sur del continente). La progresiva mejora de la esperanza de vida a lo largo de este periodo mostró dos etapas de estancamiento: al inicio del s. XX en el sur y este del continente como consecuencia de la mayor incidencia de la mortalidad infecto-contagiosa; y en la década de los sesenta para la Europa del este y central debido al aumento de las muertes por enfermedades cardiovasculares (Mackenbach, 2013). Inmediatamente después de esta segunda etapa de estancamiento se asiste a la denominada "revolución cardiovascular", caracterizada por un rápido descenso de las tasas de mortalidad por causas del aparato circulatorio y por un progresivo desplazamiento de las ganancias de años de vida a edades cada vez más avanzadas, de la que se beneficiaron en mayor medida algunos de los países del sur de Europa, lo que propició que alcanzasen, e incluso superasen, los niveles de esperanza de los países del centro y del norte del continente (Vallin y Meslé, 2004).

Partiendo de la base del conocimiento que se tiene sobre la relación entre salud autopercibida y mortalidad según edad y sexo descrito anteriormente, nuestro objetivo es el de explorar de manera descriptiva para los años 2005 y 2009 la relación entre ambos indicadores en las edades posteriores a la juventud (35-79) a nivel poblacional en diversos países europeos con trayectorias de mortalidad diferenciadas (Alemania, Francia, España, Italia, Polonia y Hungría) (Mackenbach, 2013). Nuestra idea es que en términos generales el patrón de la relación entre salud autopercibida y mortalidad debe ser común entre los diferentes países, aunque las especificidades de cada uno de ellos llevarían a encontrar ciertas diferencias según edad y sexo. De este modo, nuestras hipótesis son las siguientes:

- La falta de relación observada entre salud autopercibida y mortalidad en la población mayor apuntaría a que esta relación variaría con la edad, disminuyendo a medida que se observan edades más avanzadas

- Estos cambios con la edad en la relación entre ambos indicadores debería variar dependiendo del régimen de mortalidad de los países en las últimas décadas. De este modo, mejores valores de mortalidad deberían implicar cambios menores con la edad debido a que el proceso de reajuste de la evaluación de la salud sería más progresivo

- Diferencias entre mujeres y hombres dentro del mismo país en el patrón por edad de la relación entre ambos indicadores apuntarían a diferencias en los niveles de mortalidad y/o a diferentes contextos como referencia para evaluar la propia salud

Estas hipótesis han sido testadas mediante un análisis descriptivo de los datos agregados a nivel poblacional tanto del patrón de la prevalencia de mala salud autopercibida como de la diferencia entre las transformaciones logarítmicas de las probabilidades de morir y de la prevalencia de mala salud. Los datos proceden de la encuesta sobre Condiciones de Vida en la Unión Europea (EU- 
SILC) para la salud autopercibida, y de la Human Mortality Database (HMD) para la mortalidad.

\section{DATOS Y MÉTODO}

La Encuesta sobre Condiciones de Vida en la Unión Europea (EU-SILC) se realiza de manera homogénea en los diferentes países, permitiendo la comparativa internacional (Eurostat, 2017). La selección de los países responde tanto a criterios geográficos (sur, centro y este de Europa) como a su peso poblacional en el total de la Unión Europea (48\% del total de la población de la UE-27 en 2014). No ha sido incluido ningún país del norte del continente debido a que en estos sólo se recopiló información sobre un miembro del hogar mientras que en los países estudiados se entrevistó a todos sus miembros. Esta diferencia podría dar lugar a sesgos en los resultados, además de tener un efecto significativo en la diferencia en el número de personas entrevistadas total para cada país.

El rango de edades considerado es el de 35 a 79 años. Las edades anteriores han sido descartadas debido a la alta variabilidad observada tanto en los riesgos de morir como en los valores de salud autopercibida. En cuanto a la población de 80 años y más, no ha sido tenida en cuenta debido a que estas edades no son parte de la población objetivo de la encuesta EU-SILC, y por tanto no se tiene información. La comparación se ha realizado para mujeres y hombres de manera independiente para poder de este modo testar nuestra tercera hipótesis. La comparación de dos años (2005 y 2009) permite comprobar que los resultados no se encuentran sesgados por la posible excepcionalidad de un año concreto. Se ha escogido una distancia temporal de cuatro años para trabajar con muestras independientes, ya que este es el tiempo mínimo que se tarda en remplazar de manera completa la muestra de la EU-SILC (cada año se remplaza como mínimo un $25 \%$ de la muestra).

Los datos utilizados en el análisis se han agregado por grupos de edad quinquenales dentro del rango de edades de estudio. Por tanto, para cada país y sexo se ha trabajado con 9 observaciones (una por cada grupo quinquenal), valor insuficiente para el uso de técnicas de análisis multivariante, por lo que el análisis descriptivo se muestra como la mejor opción. Los valores sobre la prevalencia de salud autopercibida han sido obtenidos de la pregunta "¿Cómo es su salud en general? (muy buena, buena, regular, mala, muy mala)" incluida en el cuestionario de la EU-SILC. Las categorías de respuesta han sido agrupadas en dos grupos: buena salud (muy buena o buena) y mala salud (regular, mala o muy mala). La inclusión en el mismo grupo de las categorías "regular" y "mala y muy mala" se basa en la ya comentada capacidad de la salud autopercibida de informar tanto del estado del momento como de la percepción que el individuo tiene sobre la evolución reciente de su salud (Singh-Manoux, 2006). Una percepción de un estado de salud "regular" ha sido entendida como un signo de la aceleración en el proceso de deterioro, lo cual resulta especialmente relevante en las edades adultas donde la prevalencia de salud "mala" o "muy mala" no es alta, pero un 
estado "regular" indica una diferenciación significativa respecto a los iguales.

El tamaño de las muestras de EU-SILC en cada uno de los países analizados para ambos sexos asegura la consistencia de los resultados para las prevalencias de salud autopercibida. Considerando la casi nula tasa de no respuesta (siempre inferior al 2\%) para la pregunta sobre este tipo de salud, para el rango de edades entre 35 y 79 años se ha podido trabajar para el año 2005 con muestras que oscilan de los 4.444 casos para los hombres de Hungría al máximo de 16.751 mujeres para Italia. Para el año 2009 los valores varían entre 6.045 casos de nuevo para los hombres en Hungría a los 15.628 casos para las mujeres en Italia.

$\mathrm{El}$ indicador de mortalidad corresponde a las probabilidades de morir por sexo y de edad quinquenal de las tablas de mortalidad de la Human Mortality Database (HMD) para cada uno de los países explorados. Esta base forma parte de un proyecto de la University of California y del Max Planck Institute for Demografphic Research que pone a disposición de los investigadores series de tablas de mortalidad a nivel nacional para 39 países, garantizando la homogeneidad en el tratamiento de los datos de defunciones y poblaciones y en los protocolos de cálculo (Wilmoth et al., 2007), constituyendo en la actualidad el referente para la realización de estudios históricos y comparativos de mortalidad y longevidad a nivel internacional.

La comparación entre la prevalencia de mala salud autopercibida y las probabilidades de morir se ha realizado mediante la resta de sus transformaciones logarítmicas (cálculo del logaritmo en base 10 para todos los valores de ambos indicadores). Las variables resultantes de esta transformación representan los cambios relativos entre los valores de los indicadores más allá de la magnitud de los mismos. De este modo, diferencias relativas similares entre dos observaciones de distintas variables muestran valores similares en sus transformaciones logarítmicas independientemente de la magnitud original de estas variables. A partir de esta transformación, se han observado las tendencias por edad de los valores de esta resta más allá de los valores en sí, difíciles de interpretar en términos de su magnitud. Los posibles patrones que a priori se podrían encontrar son tres: un patrón estable (igualdad de la diferencia entre todos los grupos de edad quinquenales), que indicaría que la relación entre los indicadores de salud autopercibida y mortalidad resulta constante en todas las edades; un patrón ascendente, revelando que la prevalencia de mala salud aumenta con la edad con un grado superior al de la mortalidad; y finalmente, un patrón descendente con la edad, que indicaría un aumento del nivel de mortalidad con un gradiente mayor que el de la mala salud autopercibida.

Para poder medir los posibles cambios por edad en la asociación entre ambos indicadores se han calculado las rectas de ajuste sobre los patrones de la resta de los logaritmos por edad. De estas rectas nos hemos fijado tanto en el signo como en el valor de la pendiente para de este modo ver el sentido y medir la intensidad de los posibles cambios observados por edad. La capacidad de ajuste de los datos para estas rectas ha sido evaluada mediante el coeficiente de ajuste $\mathrm{R}^{2}$.

Para visualizar los resultados, los países se han agrupado según criterios geográficos: sur (España e Italia); centro (Francia y Alemania); y este (Polonia y 
Hungría). Esta agrupación coincide con las diferencias en los niveles de esperanza de vida al nacer de cada uno de los países. El primer grupo recoge los países con una mayor esperanza de vida (España (78,59 y 84,65 para hombres y mujeres respectivamente); Italia $(79,22$ y 84,24)); el segundo grupo de países presenta valores intermedios (Francia ${ }^{1}(77,78$ y 84,44); Alemania (77,46 y 82,57)); y el tercer grupo los valores más bajos así como mayores diferencias entre mujeres y hombres (Polonia (71,48 y 79,92); y Hungría $(70,21$ y 78,23)).

\section{RESULTADOS}

El presente apartado presenta dos partes diferenciadas. En la primera se describen por un lado los perfiles según edad y sexo de las prevalencias de mala salud autopercibida y de las probabilidades de morir para 2005 y 2009, así como su variación relativa entre estos dos años. Además, se muestran los valores para el indicador de exceso de prevalencia en mujeres (prevalencia de mala salud autopercibida femenina menos la misma prevalencia en los hombres, dividido todo por la prevalencia femenina) (Boerma et al., 2016). En la segunda parte se presentan los patrones por sexo de la diferencia entre los logaritmos de las prevalencias de mala salud autopercibida y las probabilidades de morir por edades quinquenales, así como las correspondientes pendientes y capacidad de ajuste de las rectas de ajuste de estos patrones.

Los patrones de la prevalencia de mala salud y de las probabilidades de morir según grandes grupos de edad (30-49, 50-64 y 65-79), sexo y país muestran tres características comunes: el lógico incremento de los valores tanto de mala salud como de mortalidad con la edad; las mayores prevalencias de mala salud para las mujeres en todos los grupos de edad, mientras que la relación es la inversa para la mortalidad; y finalmente, la mejoría de la salud y mortalidad por edad y sexo en el año 2009 respecto al 2005 (Tabla 1).

La mencionada peor salud de las mujeres respecto a los hombres presenta intensidades diferenciadas entre los países analizados. Las diferencias por sexo muestran sus menores valores en Alemania (dónde incluso las mujeres llegan a mostrar menor prevalencia de mala salud en 2005 para el grupo 35-49 y en 2009 en el grupo 50-64, aunque con valores muy pequeños, -0.5 y -0.8 puntos porcentuales respectivamente), mientras que las mayores se localizan en España e Italia (12,1 puntos porcentuales en España para el grupo 65-79 y 7,6 puntos en Italia para el grupo 50-64 en el año 2009).

En cuanto al tercer punto en común, a pesar de la mejoría en ambos indicadores entre 2005 y 2009, las variaciones porcentuales muestran diferencias cuando comparamos la salud y la mortalidad. En la mortalidad, en todos los países analizados, independientemente del sexo, las mayores variaciones se producen

${ }^{1}$ Este país se ha situado en el segundo grupo en base a los valores de su esperanza de vida al nacer para los hombres, ya que en las mujeres sus valores son muy similares a los de España e Italia 
en los grupos de edad 35-49 y 65-79. En cambio, en la mala salud, no se observa esa homogeneidad entre países y sexos. En Alemania y Francia el patrón de salud muestra la misma variación que el de la mortalidad, dándose en ambos sexos los mayores incrementos en los grupos 35-49 y 65-79 años. En el resto de países son los grupos de edad 35-49 y 50-64 los que muestran unas mayores mejorías relativas en ambos sexos, con la excepción de las mujeres españolas, donde se localizan en los grupos de 50-64 y 65-79 años.

Tabla 1. Prevalencia de mala salud autopercibida y probabilidades de morir (\%o) y variación porcentual por grupos de edad y sexo. 2005 y 2009

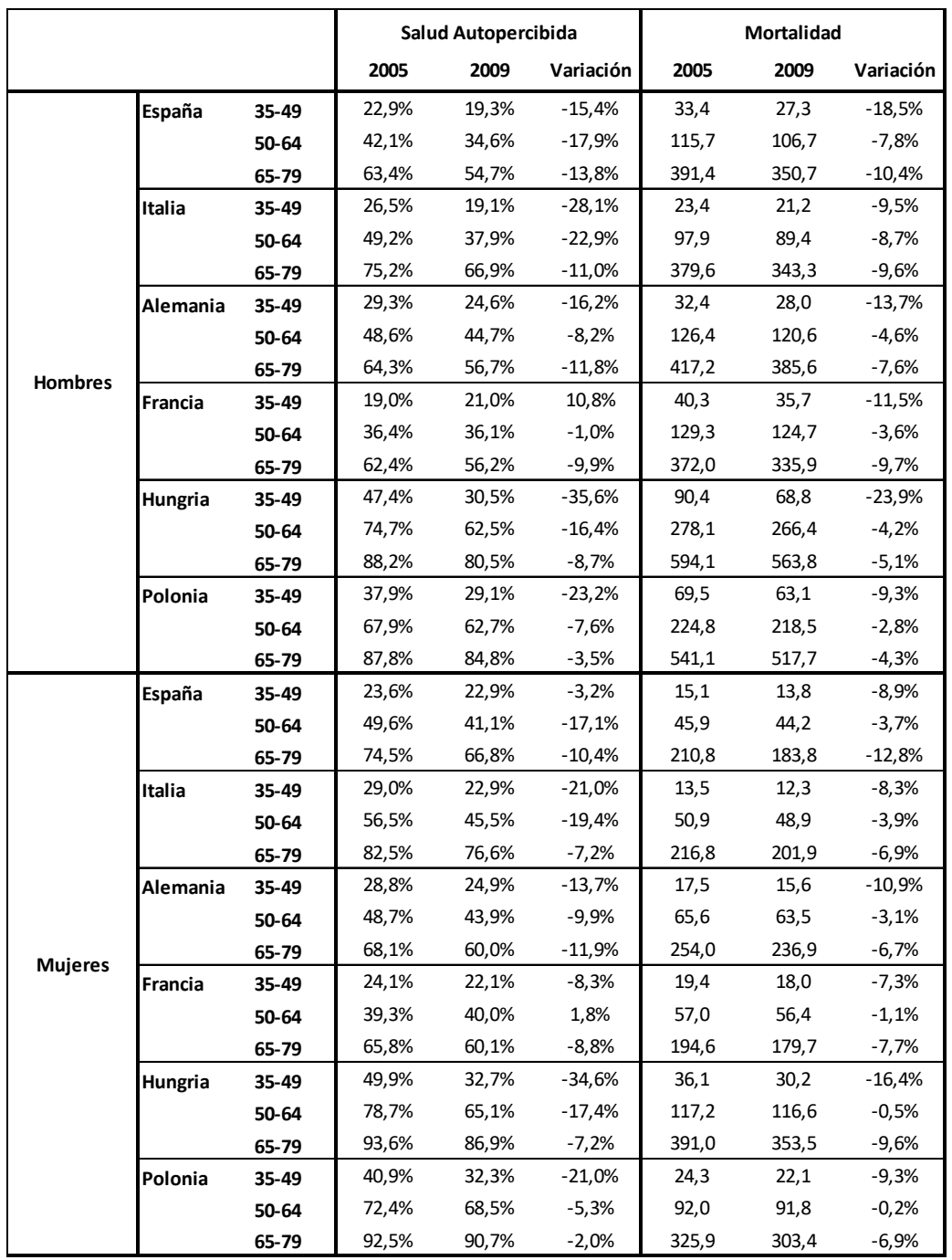

Fuente: Elaboración propia a partir de la EU-SILC y HMD. 
Las prevalencias de mala salud también muestran diferencias entre países. Se observa cómo los países del este de Europa (Hungría y Polonia) presentan valores más altos de mala salud en ambos años independientemente del sexo. De hecho, se trata de los únicos países con prevalencias de mala salud superiores al $80 \%$ en el grupo de mayores en ambos sexos en los dos años. En el otro extremo, Alemania, Francia y España muestran menores prevalencias, mientras que Italia se sitúa en una posición intermedia, pero con valores más cercanos a los de los países con baja prevalencia en mala salud.

Los resultados presentados en la Tabla 2 dan información más detallada sobre la mayor prevalencia de mala salud en las mujeres respecto de los hombres por grupos de edad para cada país y año observados. Para todos los países, con las únicas excepciones de Alemania y Francia, aunque sólo para el grupo de edades 35-49, se observa un incremento en las diferencias en las prevalencias de mala salud autopercibida entre mujeres y hombres de manera casi sistemática en todos los grupos de edad. En cuanto a las diferencias entre los países analizados, se puede ver como aquellos con esperanzas de vida superiores, sobre todo en el caso femenino, como son España e Italia, muestran mayores diferencias entre mujeres y hombres, con la única excepción del grupo de 35-49 en España. En cuanto a las menores diferencias se observan en Hungría y Alemania, destacando este último país por su casi nula diferencia en la salud autopercibida de mujeres y hombres en edades más jóvenes de 65 años.

Tabla 2. Indicador de exceso de prevalencia de mala salud autopercibida en mujeres. 2005 y 2009

\begin{tabular}{|ll|cc|}
\hline & & \multicolumn{2}{|c|}{ Salud Autopercibida } \\
& & $\mathbf{2 0 0 5}$ & $\mathbf{2 0 0 9}$ \\
\hline España & $\mathbf{3 5 - 4 9}$ & $3,2 \%$ & $15,4 \%$ \\
& $\mathbf{5 0 - 6 4}$ & $15,0 \%$ & $15,9 \%$ \\
& $\mathbf{6 5 - 7 9}$ & $14,8 \%$ & $18,1 \%$ \\
\hline Italia & $\mathbf{3 5 - 4 9}$ & $8,6 \%$ & $16,9 \%$ \\
& $\mathbf{5 0 - 6 4}$ & $12,9 \%$ & $16,7 \%$ \\
& $\mathbf{6 5 - 7 9}$ & $8,8 \%$ & $12,6 \%$ \\
\hline Alemania & $\mathbf{3 5 - 4 9}$ & $-1,8 \%$ & $1,1 \%$ \\
& $\mathbf{5 0 - 6 4}$ & $0,1 \%$ & $-1,8 \%$ \\
& $\mathbf{6 5 - 7 9}$ & $5,7 \%$ & $5,5 \%$ \\
\hline Francia & $\mathbf{3 5 - 4 9}$ & $21,2 \%$ & $4,8 \%$ \\
& $\mathbf{5 0 - 6 4}$ & $7,4 \%$ & $10,0 \%$ \\
& $\mathbf{6 5 - 7 9}$ & $5,3 \%$ & $6,5 \%$ \\
\hline Hungria & $\mathbf{3 5 - 4 9}$ & $5,1 \%$ & $6,6 \%$ \\
& $\mathbf{5 0 - 6 4}$ & $5,1 \%$ & $4,0 \%$ \\
& $\mathbf{6 5 - 7 9}$ & $5,8 \%$ & $7,3 \%$ \\
\hline Polonia & $\mathbf{3 5 - 4 9}$ & $7,2 \%$ & $9,8 \%$ \\
& $\mathbf{5 0 - 6 4}$ & $6,2 \%$ & $8,5 \%$ \\
& $\mathbf{6 5 - 7 9}$ & $5,0 \%$ & $6,5 \%$ \\
\hline
\end{tabular}

Fuente: Elaboración propia a partir de la EU-SILC. 
Respecto de la relación entre la prevalencia de la mala salud percibida y los niveles de mortalidad, la representación gráfica de la diferencia entre la transformada logarítmica de ambas variables muestra un mismo patrón para todos los países independientemente del sexo y del año, ya que sus valores disminuyen a medida que aumenta la edad (Figuras 1). Por tanto, este patrón nos indica que el nivel de mortalidad por edad aumenta con un gradiente mayor que el de la mala salud autopercibida, coincidiendo con estudios anteriores (Helweg-Larsen et al., 2003). Este hecho parece darse de manera constante en ambos años tanto en la tendencia como en la intensidad de los valores del indicador. Sin embargo, esta semejanza es mayor en los grupos de edad más avanzados mientras que en las primeras edades adultas se observan mayores diferencias, siendo más visibles en ambos sexos en Hungría e Italia, en los hombres franceses y en las mujeres alemanas. En cuanto a las diferencias entre sexos, las mujeres muestran valores mayores en la diferencia entre ambos factores en todas las edades debido a sus mayores prevalencias de mala salud y menores probabilidades de morir.

Los patrones de los distintos países muestran valores más altos para el indicador en Francia, Italia y España, tanto en hombres como en mujeres. Estas diferencias en la magnitud del indicador se deben a su menor mortalidad como confirman sus mayores esperanzas de vida. Para el resto de países, las diferencias son menores, siendo más pequeñas cuanto menor es la esperanza de vida al nacer (Polonia y Hungría).

\section{Grupo de Figuras 1. Diferencia entre los logaritmos de la prevalencia de mala salud autopercibida y de las probabilidades de morir por edades quinquenales y sexo. 2005 y 2009}

\section{España}

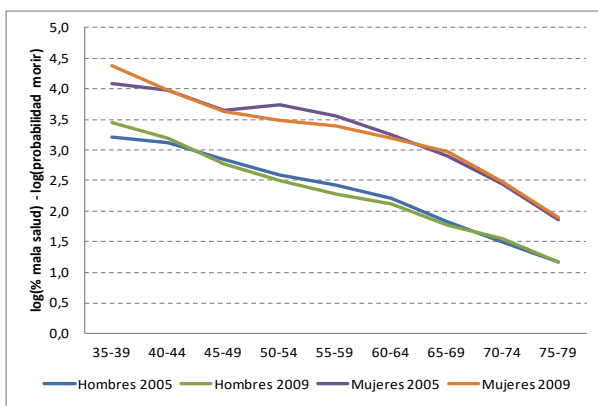

Italia

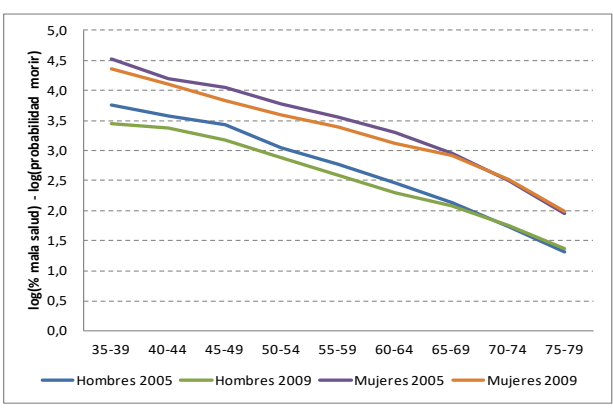


Alemania

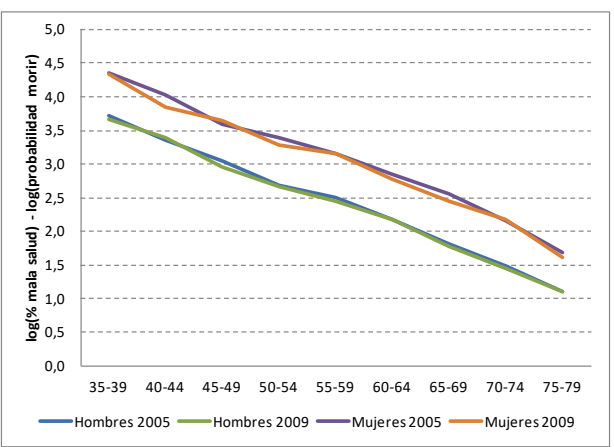

Polonia

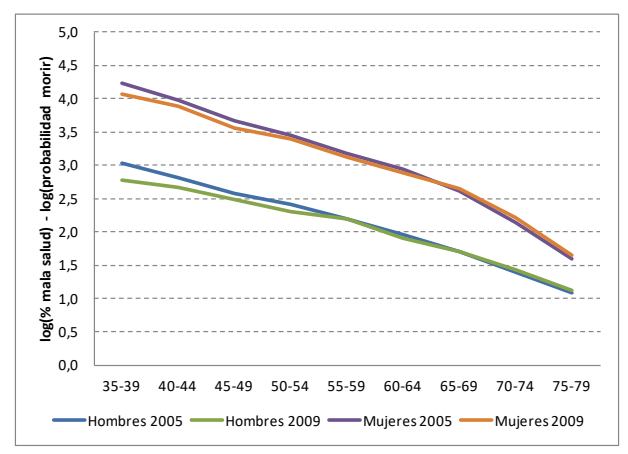

Francia

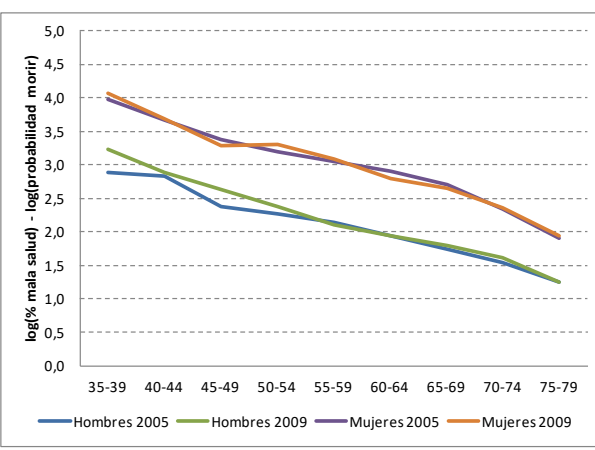

Hungría

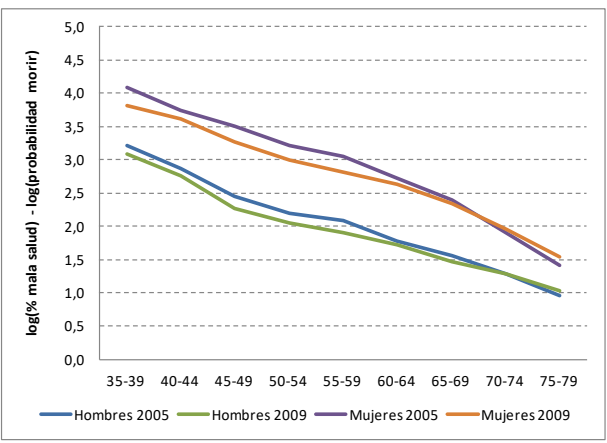

Fuente: Elaboración propia a partir de datos de la EU-SILC y HMD para 2005 y 2009

Por último, la Tabla 3 muestra los resultados para la pendiente y el coeficiente de ajuste $\left(\mathrm{R}^{2}\right)$ de las rectas de tendencia lineal entre salud autopercibida y mortalidad para cada de país, año y sexo. Los valores de $\mathrm{R}^{2}$ son muy elevados, confirmando la linealidad de la relación entre mala salud y mortalidad por edad. Cabe destacar el caso de España, que aunque sin dejar de mostrar valores altos para la $\mathrm{R}^{2}$, estos son menores para las mujeres respecto al resto de países. En cuanto a las pendientes de estas rectas de ajuste, todas presentan un signo negativo, indicando un descenso en la relación entre ambos indicadores como consecuencia de un mayor aumento con la edad del logaritmo de las probabilidades de morir respecto del logaritmo de la prevalencia de mala salud autopercibida. Cuando se comparan los resultados entre los diferentes países vemos como Alemania, para ambos sexos, muestra mayores valores en ambos años, mientras que Francia los menores. En cuanto a las diferencias entre mujeres y hombres dentro de cada país, los países del sur y del centro del continente muestran diferencias nulas o muy pequeñas, mientras que en los países del este estas diferencias son 
mayores, siendo el descenso por edad de la relación entre ambos indicadores mayor entre las mujeres.

Tabla 3. Coeficientes de las líneas de tendencia para la resta entre el logaritmo de la mala salud autopercibida y el logaritmo de las probabilidades de morir por edades quinquenales y sexo para cada país. 2005 y 2009

\begin{tabular}{|c|c|c|c|c|c|}
\hline \multicolumn{2}{|c}{} & \multicolumn{2}{c|}{2005} & \multicolumn{2}{c|}{2009} \\
\hline \multirow{3}{*}{ España } & Pendiente & $\mathbf{R}^{\mathbf{2}}$ & Pendiente & $\mathbf{R}^{\mathbf{2}}$ \\
\cline { 2 - 6 } & Hombres & $-0,26$ & 0,98 & $-0,27$ & 0,99 \\
\hline \multirow{3}{*}{ Italia } & Mujeres & $-0,26$ & 0,90 & $-0,26$ & 0,90 \\
\cline { 2 - 6 } & Mumbres & $-0,31$ & 0,99 & $-0,27$ & 0,99 \\
\hline \multirow{3}{*}{ Alemania } & Hombres & $-0,30$ & 0,97 & $-0,28$ & 0,98 \\
\cline { 2 - 6 } & Mujeres & $-0,32$ & 0,99 & $-0,31$ & 0,99 \\
\hline \multirow{3}{*}{ Francia } & Hombres & $-0,20$ & 0,98 & $-0,23$ & 0,99 \\
\cline { 2 - 6 } & Mujeres & $-0,23$ & 0,97 & $-0,24$ & 0,97 \\
\hline \multirow{2}{*}{ Polonia } & Hombres & $-0,24$ & 0,99 & $-0,20$ & 0,98 \\
\cline { 2 - 6 } & Mujeres & $-0,31$ & 0,98 & $-0,28$ & 0,97 \\
\hline \multirow{2}{*}{ Hungría } & Hombres & $-0,27$ & 0,99 & $-0,24$ & 0,97 \\
\cline { 2 - 6 } & Mujeres & $-0,31$ & 0,98 & $-0,27$ & 0,99 \\
\hline
\end{tabular}

Fuente: Elaboración propia

\section{DISCUSIÓN}

El trabajo describe a nivel poblacional la relación formal entre el indicador de la salud autopercibida y las probabilidades de morir en edades posteriores a la juventud (35-79) según grupos de edad quinquenales en seis países del ámbito europeo (Alemania, Francia, España, Italia, Polonia y Hungría), en los años 2005 y 2009. Los datos proceden de la encuesta sobre Condiciones de Vida en la Unión Europea (EU-SILC) para la salud autopercibida, y de la Human Mortality Database (HMD) para la mortalidad. Se han observado patrones claros por edad y sexo comunes en los seis países estudiados, aunque también existen diferencias claras que se comentan a continuación.

Los resultados permiten dar luz sobre las tres hipótesis planteadas en un inicio. En cuanto a la primera, el posible cambio en la relación formal entre ambos indicadores con la edad, nuestros resultados muestran que aunque el patrón de ambos indicadores individualmente sea creciente, el valor relativo de este incremento no es igual para la mortalidad y para la mala salud. De hecho, en el caso concreto de la prevalencia de mala salud el patrón de crecimiento con la edad parece reducirse o incluso estancarse a medida que se observan edades mayores, 
siendo este resultado común para ambos sexos en todos los países analizados. Una explicación sería el posible efecto de selección por supervivencia derivado de la comparación entre indicadores calculados con información de toda la población (mortalidad) o en base a muestras representativas (salud autopercibida). Esta selección por supervivencia se basaría en el hecho que la participación en una encuesta requiere de unas ciertas capacidades por parte del individuo claramente vinculadas con su salud, y que, por tanto, en las edades más avanzadas el porcentaje de personas entrevistadas con un buen estado de salud podría ser superior al porcentaje real en el total poblacional. A esto, debe añadirse que la población institucionalizada no es objeto de las muestras de la encuesta EUSILC. Sin embargo, trabajos recientes realizados a partir del seguimiento de los mismos individuos a lo largo de un periodo de 15 años, en los cuales se comparó la supervivencia y la salud autopercibida en el contexto de los Estados Unidos, muestran un resultado similar en cuanto al incremento diferenciado de ambos indicadores según la edad. De este modo, además de descartar la selección de las personas con un mejor perfil de salud como razón más importante de los resultados encontrados (Woo y Zajacova, 2014), la similitud con los de este trabajo indicaría que el hecho de trabajar únicamente con datos de naturaleza transversal no introduce un sesgo significativo.

Una vez descartado el efecto de la selección por supervivencia, ganaría peso la ya apuntada normalización por parte del individuo de la propia morbilidad (Singh-Manoux, 2006; Oksuzyan et al., 2009) como explicación al menor incremento de la prevalencia de mala salud entre edades. Esta normalización se basaría en dos de los tres contextos propuestos por Jylhä (2009) que influyen sobre la percepción individual de la salud: la biografía de salud del individuo y los grupos de referencia para su evaluación. En cuanto al primero, teniendo en cuenta que la salud autopercibida informa sobre la evolución reciente de la salud del individuo (Singh-Manoux, 2006), serían los momentos de cambio los que sobre todo afectan a la percepción del individuo. Mientras que una vez se produce un estancamiento de la evolución de la salud, incluso sea en base a una situación de mala salud (enfermedades crónicas, discapacidades, etc), la percepción del individuo se adaptaría a su estado de salud. En cuanto al grupo de referencia para su evaluación, el mayor peso con la edad de los perfiles de mala salud llevaría a incrementar el umbral entre percepción positiva o negativa de la propia salud al compararse con sus homólogos. De todos modos, el hecho de no poder trabajar con poblaciones más allá de los 79 años implica que nuestra hipótesis primera se debería corroborar mediante con datos para la salud autopercibida y la mortalidad en edades de 80 años y más.

La segunda de las hipótesis planteaba que la relación por edad entre ambos indicadores debería variar dependiendo del régimen de mortalidad de los países en las últimas décadas. El país que muestra en ambos años un mayor cambio con la edad de la relación entre salud autopercibida y mortalidad es Alemania. Se trata de un país con una particularidad singular como consecuencia de la reunificación que sigue mostrando dos perfiles de morbilidad diferenciados en su parte este y oeste (Müller-Nordhorn, 2004). Esto último podría ir en la direc- 
ción de nuestra hipótesis, aunque el hecho de que aquellos países con esperanzas de vida superiores como son España e Italia no muestren menores magnitudes para el cambio en la asociación entre ambos indicadores por edad nos disuade de confirmar nuestra hipótesis. Por tanto, los resultados parecen apuntar a que entre las características propias de cada país que determinan la respuesta de los individuos al ser preguntados por su propia salud (Jylhä, 2009), los factores culturales singulares relacionados con los hábitos y comportamientos saludables, así como con la propia declaración de la salud (Olsen y Dahl, 2007) tendrían un mayor peso para entender las diferencias entre países, mientras que los efectos de los diferentes regímenes de mortalidad de cada país sólo serían visibles en situaciones atípicas como el caso alemán.

La tercera de las hipótesis proponía que diferencias entre mujeres y hombres del mismo país en el cambio de la relación entre ambos indicadores con la edad apuntarían a diferencias en los niveles de mortalidad y/o a diferentes contextos como referencia para evaluar la propia salud. Los resultados según sexo muestran valores más altos de mala salud entre las mujeres en todos los países mientras que en la mortalidad se da el resultado inverso, confirmando la "paradoja de salud y mortalidad" entre sexos (Oksuzyan et al., 2009). Aunque como se ha apuntado anteriormente esta paradoja fue inicialmente explicada a partir de las diferencias en los perfiles de morbilidad por sexo (Oksuzyan et al., 2008; Oksuzyan et al., 2010), trabajos posteriores han mostrado que una vez se controla por diversos determinantes sociales de la salud además de por indicadores de salud objetiva, la mayor prevalencia de la mala salud autopercibida entre las mujeres respecto de los hombres desaparece o incluso se invierte (Crimmins et al., 2010; Gumà et al., 2015). Esto apunta a la combinación del perfil de morbilidad femenino y las desigualdades de género existentes aún en las sociedades occidentales como explicación para entender la diferencia en la salud de mujeres y hombres. Nuestros resultados señalan a los países del este, Polonia y Hungría, como los únicos casos en los cuales se observan diferencias entre mujeres y hombres en el cambio de la relación entre salud autopercibida y mortalidad con la edad, siendo estos cambios más acentuados entre ellas. Precisamente son estos países los que muestran mayores niveles de desigualdad de género cuando se toma el Gender Equality Inex (European Institute for Gender Equality, 2017) como referencia. Este indicador toma una escala de valores de 0 a 100 , siendo los valores más altos indicativos de un mayor nivel de igualdad. Mientras que los cuatro países del centro y sur de Europa presentan valores superiores a 60 en cada caso para el año 2015, Polonia y Hungría estaban por debajo de este valor (56,8 y 50,8 respectivamente). Por tanto, nuestra tercera y última hipótesis parece ir en la dirección correcta a la vista de nuestros resultados, aunque sería necesario un estudio con un mayor número de países para poder confirmar su certeza.

Además, el hecho de que todos los países muestren mejores valores en su salud autopercibida cuatro años más tarde apuntaría en la dirección de una contracción de la morbilidad en un contexto de incremento de la esperanza de vida o, como mínimo, una evolución relativamente paralela entre las ganancias 
de longevidad y de salud, es decir aquella que se define como una situación de "equilibrio dinámico" (Manton, 1982). Este aspecto debería ser analizado con más detalle en el futuro, sirviendo para actualizar nuestro conocimiento sobre las diferencias en los perfiles de salud entre los países europeos. Los resultados sobre la prevalencia de mala salud por edad y sexo no corroboran completamente las conclusiones de trabajos previos sobre la diferenciación entre países del sur, este y centro de Europa, en los cuales se apuntaban peores valores de salud autopercibida entre los primeros a pesar de su mayor esperanza de vida (Knesebeck et al., 2006; Olsen y Dahl, 2007). Por un lado, España presenta prevalencias de mala salud muy similares a las de Francia y Alemania, mientras Italia se muestra como un caso intermedio entre estos últimos y los países del este, apuntando que los países del sur de Europa parecen converger con los del centro.

\section{CONCLUSIONES}

Los resultados muestran la existencia de un patrón similar entre países en los perfiles de salud autopercibida y mortalidad que se mantiene en el tiempo. La mejora de la salud autopercibida con el tiempo paralela a los avances continuados en la mortalidad apuntaría en la dirección de una relación formal entre ambos indicadores, aunque esta relación disminuye a medida que avanza la edad, y muestra diferencias entre mujeres y hombres dependiendo del contexto específico de cada país.

\section{BIBLIOGRAFÍA}

Benyamini Y, Leventhal E, Leventhal H, Elderly people's ratings of the importance of health-related factors to their self-assessments of health. Social Science \& Medicine. 2003; 56: 1661-1667.

Boerma T, Hosseinpoor AR, Verdes E, Chatterji S. A global assessment of the gender gap in self-reported health with survey data from 59 countries. BMC Public Health. 2016 16: 675

Carlson P,. Self-perceived health in East and West Europe: another European health divide. Social Science \& Medicine. 1998; 46: 1355-1366.

Crimmins E. M, Kim J. K,. Solé-Auró A. Gender differences in health: results from SHARE, ELSA and HRS. European journal of public health. 2010; 21.1: 81-91

Eikemo T. A., Bambra C, Judge K, et al. Welfare state regimes and differences in selfperceived health in Europe: a multilevel analysis. Social science \& medicine. 2008; 66: 2281-2295.

European Institute for Gender Equality. Gender Equality Index Score-2015. Consulta el 7 de Diciembre 2017. (http://eige.europa.eu/gender-equality-index/2015)

Eurostat. EU statistics on income and living conditions (EU-SILC) methodology. Consulta el 7 de Diciembre 2017.(http://ec.europa.eu/eurostat/statistics-explained/index. php/EU_statistics_on_income_and_living_conditions_(EU-SILC)_methodology)

Franks P, Gold M. R., Fiscella K,. Sociodemographics, self-rated health, and mortality in the US. Social science \& medicine. 2003; 56: 2505-2514. 
Goldsheider F. K. Men, children and the future of the family in the third millennium. The Futurist. 2000; 32: 527-538.

Gumà J, Cámara A. D. ¿Informa la salud autopercibida sobre las condiciones objetivas de salud? Algunas conclusiones a partir del análisis demográfico de microdatos de la Encuesta Nacional de Salud. Revista Estadística española. 2014; 56: 61-76.

Gumà J, Treviño R, Cámara A. D. Posición en el hogar y género. Desigualdades en la calidad de vida relacionada con la salud entre la población adulta en España. Revista Internacional de Sociología. 2015; 73, 103989-ris.

Helweg-Larsen M, Kjøller M, Thoning H. Do age and social relations moderate the relationship between self-rated health and mortality among adult Danes? Social Science \& Medicine 2003; 57: 1237-47.

Idler E, Benyamini Y,. Self-rated health and mortality: A review of twenty-seven community studies. Journal of Health \& Social Behavior. 1997; 38: 21-37.

Idler, E. L. Gender differences in self-rated health, in mortality, and in the relationship between the two. Gerontologist. 2003; 43: 372-375.

Jylhä M,. What is self-rated health and why does it predict mortality? Towards a unified conceptual model. Social science \& medicine. 2009; 69: 307-316.

Knesebeck O, Verde P, Dragano N,. Education and health in 22 European countries. Social Science \& Medicine. 2006; 63: 1344-1351.

Manton, K.G., Changing concepts of morbidity and mortality in the elderly population. The Milbank Memorial Fund Quarterly. Health and Society. 1982; 60; 183-244.

Mackenbach J.P. Convergence and divergence of life expectancy in Europe: a centennial view European Journal of Epidemiology. 2013; 28: 229-240.

Marmot M,. Social determinants of health inequalities. The Lancet. 2005 365: 10991104.

McDonald P,. Gender equity in theories of fertility transition. Population and Development Review. 2000; 26(3): 427-440.

Norris C. M, Murray J. W, Triplett L. S. et al. Gender roles in persistent sex differences in health-related quality-of-life outcomes of patients with coronary artery disease. Gender medicine. 2010; 7: 330-339.

Tamayo-Fonseca N, Quesada J. A, Nolasco A. et al. Self-rated health and mortality: a follow-up study of a Spanish population. Public health. 2013 127: 1097-1104.

Mossey J, Shapiro E,. Self-rated health: a predictor of mortality among the elderly. American Journal of Public Health. 1982; 72: 800-08.

Müller-Nordhorn J, Rossnagel K, Mey W, Willich SN. Regional variation and time trends in mortality from ischaemic heart disease: East and West Germany 10 years after reunification. Journal of Epidemiology and Community Health. 2004; 58: 481485

Oksuzyan A, Juel K, Vaupel J, et al. Men: good health and high mortality. Sex differences in health and aging. Aging Clinical and Experimental Research. 2008; 20: 91-102.

Oksuzyan A, Petersen I, Stovring H, et al. The male-female health-survival paradox: A survey and register study of the impact of sex-specific selection and information bias. Annals of Epidemiology. 2009; 19: 504-511.

Oksuzyan A, Gumà J, Doblhammer G. 2018. Sex differences in health and survival. En: A Demographic Perspective on Gender, Family and Health in Europe. Holanda: Springer. 2018; p. 65-103.

Olsen K, Dahl S,. Health differences between European countries. Social Science \& Medicine. 2007; 64: 1665-1678. 
Organización Mundial de la Salud (OMS). A conceptual framework for action on the social determinants of health. Comission on social determinants of health. 2007; Consulta 15 de Septiembre 2015

(http://www.who.int/social determinants/resources/csdh framework action 05 07.pdf)

Pongiglione B, Sabater A. The Role of Education at Young and Older Ages in Explaining Health Inequalities in Europe. Population, Space and Place. 2016; 22: 255-275.

Quesnel-Vallée A. Self-rated health: caught in the crossfire of the quest for 'true'health?. International Journal of Epidemiology. 2007; 36: 1161-1164.

Sanderson W, Scherbov S. Gender Inequality in Survival at Older Ages. IIASA Working Paper. 2017. IIASA, Laxenburg, Austria: WP-17-002

Singh-Manoux A, Martikainen P, Ferrie J, Zins M, Marmot M, Goldberg M. What does self rated health measure? Results from the British Whitehall II and French Gazel cohort studies. Journal of Epidemiology \& Community Health. 2006; 60: 364-372.

Vallin J, Meslé F, Valkonen T,. Tendences en matière de mortalité et mortalité différentielle, Etudes démographiques, Editions du Conseil de l’Europe. 2001; 36

Vallin J, Meslé F. Convergences and divergences in mortality. A new approach to health transition. Demographic Research. 2004; 1-2: 12-43

Wilmoth F, Andreev K, Jdanov D et al. Methods Protocol for the Human Mortality Database. 2007. 1-80.

(http://www.mortality.org/Public/Docs/MethodsProtocol.pdf)

Woo H, Zajacova A,. Why Self-Rated Health Predicts Mortality Less Well at Older Ages: Physical and Mental Health Correlates of Self-Rated Health. Libro de ponencias de la European Population Conference. 25-28 de Junio. 2014; Budapest, Hungría. 


\section{Anexo}

Tabla A1. Perfil muestra EU-SILC según edad, sexo y país. 2005 y 2009

\begin{tabular}{|c|c|c|c|c|c|c|c|}
\hline & \multicolumn{3}{|c|}{2005} & \multicolumn{3}{|c|}{2009} \\
\hline & & Hombres & Mujeres & Total & Hombres & Mujeres & Total \\
\hline \multirow{10}{*}{ España } & $35-39$ & 1283 & 1427 & 2710 & 1283 & 1429 & 2712 \\
\hline & $40-44$ & 1405 & 1493 & 2898 & 1420 & 1470 & 2890 \\
\hline & $45-49$ & 1292 & 1398 & 2690 & 1389 & 1485 & 2874 \\
\hline & 50-54 & 1111 & 1177 & 2288 & 1293 & 1360 & 2653 \\
\hline & $55-59$ & 1108 & 1202 & 2310 & 1030 & 1135 & 2165 \\
\hline & $60-64$ & 978 & 1021 & 1999 & 1089 & 1178 & 2267 \\
\hline & $65-69$ & 845 & 925 & 1770 & 879 & 1025 & 1904 \\
\hline & $70-74$ & 804 & 998 & 1802 & 748 & 880 & 1628 \\
\hline & $75-79$ & 894 & 1229 & 2123 & 700 & 860 & 1560 \\
\hline & Total & 9720 & 10870 & 20590 & 9831 & 10822 & 20653 \\
\hline \multirow{10}{*}{ Italia } & $35-39$ & 2174 & 2260 & 4434 & 1811 & 1952 & 3763 \\
\hline & $40-44$ & 2155 & 2274 & 4429 & 2006 & 2142 & 4148 \\
\hline & $45-49$ & 1981 & 1982 & 3963 & 1966 & 1991 & 3957 \\
\hline & $50-54$ & 1798 & 1888 & 3686 & 1756 & 1818 & 3574 \\
\hline & $55-59$ & 1970 & 2047 & 4017 & 1580 & 1678 & 3258 \\
\hline & $60-64$ & 1545 & 1638 & 3183 & 1596 & 1683 & 3279 \\
\hline & $65-69$ & 1615 & 1749 & 3364 & 1417 & 1546 & 2963 \\
\hline & $70-74$ & 1278 & 1508 & 2786 & 1330 & 1557 & 2887 \\
\hline & $75-79$ & 1039 & 1405 & 2444 & 1051 & 1261 & 2312 \\
\hline & Total & 15555 & 16751 & 32306 & 14513 & 15628 & 30141 \\
\hline \multirow{10}{*}{ Alemania } & $35-39$ & 938 & 1336 & 2274 & 765 & 882 & 1647 \\
\hline & $40-44$ & 1215 & 1681 & 2896 & 1120 & 1260 & 2380 \\
\hline & $45-49$ & 1173 & 1527 & 2700 & 1168 & 1270 & 2438 \\
\hline & $50-54$ & 1113 & 1222 & 2335 & 1030 & 1156 & 2186 \\
\hline & $55-59$ & 912 & 1072 & 1984 & 987 & 1132 & 2119 \\
\hline & $60-64$ & 957 & 1042 & 1999 & 908 & 1048 & 1956 \\
\hline & $65-69$ & 1087 & 1229 & 2316 & 1243 & 1313 & 2556 \\
\hline & $70-74$ & 681 & 764 & 1445 & 1067 & 1064 & 2131 \\
\hline & $75-79$ & 453 & 518 & 971 & 558 & 463 & 1021 \\
\hline & Total & 8529 & 10391 & 18920 & 8846 & 9588 & 18434 \\
\hline \multirow{10}{*}{ Francia } & $35-39$ & 822 & 890 & 1712 & 867 & 881 & 1748 \\
\hline & $40-44$ & 879 & 950 & 1829 & 864 & 965 & 1829 \\
\hline & $45-49$ & 799 & 893 & 1692 & 861 & 982 & 1843 \\
\hline & $50-54$ & 817 & 850 & 1667 & 790 & 920 & 1710 \\
\hline & 55-59 & 811 & 825 & 1636 & 891 & 905 & 1796 \\
\hline & $60-64$ & 576 & 610 & 1186 & 747 & 856 & 1603 \\
\hline & $65-69$ & 457 & 557 & 1014 & 543 & 605 & 1148 \\
\hline & $70-74$ & 462 & 542 & 1004 & 512 & 514 & 1026 \\
\hline & $75-79$ & 490 & 648 & 1138 & 402 & 546 & 948 \\
\hline & Total & 6113 & 6765 & 12878 & 6477 & 7174 & 13651 \\
\hline
\end{tabular}




\begin{tabular}{|c|c|c|c|c|c|c|c|}
\hline & \multicolumn{3}{|c|}{2005} & \multicolumn{3}{|c|}{2009} \\
\hline & & Hombres & Mujeres & Total & Hombres & Mujeres & Total \\
\hline \multirow{10}{*}{ Hungria } & $35-39$ & 608 & 569 & 1177 & 785 & 882 & 1667 \\
\hline & $40-44$ & 516 & 525 & 1041 & 798 & 869 & 1667 \\
\hline & $45-49$ & 611 & 651 & 1262 & 749 & 842 & 1591 \\
\hline & 50-54 & 668 & 760 & 1428 & 937 & 1148 & 2085 \\
\hline & $55-59$ & 512 & 627 & 1139 & 854 & 1105 & 1959 \\
\hline & $60-64$ & 482 & 641 & 1123 & 711 & 949 & 1660 \\
\hline & $65-69$ & 367 & 622 & 989 & 545 & 787 & 1332 \\
\hline & $70-74$ & 348 & 533 & 881 & 373 & 697 & 1070 \\
\hline & $75-79$ & 332 & 652 & 984 & 293 & 565 & 858 \\
\hline & Total & 4444 & 5580 & 10024 & 6045 & 7844 & 13889 \\
\hline \multirow{10}{*}{ Polonia } & $35-39$ & 1356 & 1415 & 2771 & 1136 & 1172 & 2308 \\
\hline & $40-44$ & 1491 & 1649 & 3140 & 1144 & 1205 & 2349 \\
\hline & $45-49$ & 1797 & 2026 & 3823 & 1323 & 1421 & 2744 \\
\hline & $50-54$ & 1807 & 2043 & 3850 & 1515 & 1669 & 3184 \\
\hline & $55-59$ & 1472 & 1611 & 3083 & 1413 & 1593 & 3006 \\
\hline & $60-64$ & 866 & 1084 & 1950 & 1065 & 1289 & 2354 \\
\hline & $65-69$ & 880 & 1191 & 2071 & 714 & 930 & 1644 \\
\hline & $70-74$ & 772 & 1069 & 1841 & 631 & 964 & 1595 \\
\hline & $75-79$ & 694 & 1169 & 1863 & 531 & 815 & 1346 \\
\hline & Total & 11135 & 13257 & 24392 & 9472 & 11058 & 20530 \\
\hline
\end{tabular}

Fuente: Elaboración propia a partir de datos de la EU-SILC para 2005 y 2009 